\title{
Fluorescence life-time imaging microscopy (FLIM) monitors tumor cell death triggered by photothermal therapy with $\mathrm{MoS}_{2}$ nanosheets
}

\author{
Hongda Liang ${ }^{\dagger}$, Zheng Peng ${ }^{\dagger}$, Xiao Peng*, Yufeng Yuan, \\ Teng Ma, Yiwan Song, Jun Song and Junle Qu \\ Key Laboratory of Optoelectronic Devices and \\ Systems of Ministry of Education and Guangdong Province \\ College of Physics and Optoelectronic Engineering \\ Shenzhen University, Shenzhen 518060, P. R. China \\ *pengxiao_px@szu.edu.cn
}

Received 14 March 2019

Accepted 6 May 2019

Published 26 June 2019

\begin{abstract}
Recently, photothermal therapy (PTT) has been proved to have great potential in tumor therapy. In the last several years, $\mathrm{MoS}_{2}$, as one novel member of nanomaterials, has been applied into PTT due to its excellent photothermal conversion efficacy. In this work, we applied fluorescence lifetime imaging microscopy (FLIM) techniques into monitoring the PPTtriggered cell death under $\mathrm{MoS}_{2}$ nanosheet treatment. Two types of $\mathrm{MoS}_{2}$ nanosheets (single layer nanosheets and few layer nanosheets) were obtained, both of which exhibited presentable photothermal conversion efficacy, leading to high cell death rates of 4T1 cells (mouse breast cancer cells) under PTT. Next, live cell images of $4 \mathrm{~T} 1$ cells were obtained via directly labeling the mitochondria with Rodamine123, which were then continuously observed with FLIM technique. FLIM data showed that the fluorescence lifetimes of mitochondria targeting dye in cells treated with each type of $\mathrm{MoS}_{2}$ nanosheets significantly increased during PTT treatment. By contrast, the fluorescence lifetime of the same dye in control cells (without nanomaterials) remained constant after laser irradiation. These findings suggest that FLIM can be of great value in monitoring cell death process during PTT of cancer cells, which could provide dynamic data of the cellular microenvironment at single cell level in multiple biomedical applications.
\end{abstract}

Keywords: Fluorescence lifetime imaging microscopy (FLIM); $\mathrm{MoS}_{2}$ nanosheets; photothermal therapy (PTT); 4T1 cells.

\footnotetext{
${ }^{*}$ Corresponding author.

$\dagger$ Hongda Liang and Zheng Peng contributed equally to this work and are considered as co-first authors.
}

This is an Open Access article published by World Scientific Publishing Company. It is distributed under the terms of the Creative Commons Attribution 4.0 (CC-BY) License. Further distribution of this work is permitted, provided the original work is properly cited. 


\section{Introduction}

At present, cancer is one of most lethal diseases in the world. There are still great limitations of traditional treatment strategies including surgery, chemotherapy, and radiotherapy, leading to development of a lot of alternative novel therapeutic approaches. ${ }^{1-3}$ Among these various treatment approaches, photothermal therapy (PTT), which employs heat generated from visible or near infrared (NIR) absorbed materials to ablate tumors, has attracted more and more attention due to its advantages including minimal invasiveness and high efficiency against drug-resistant tumors. ${ }^{4-8}$ In the last several years, two-dimensional (2D) molybdenum disulfide $\left(\mathrm{MoS}_{2}\right)$ nanomaterials have shown high light-absorption ability and satisfactory biocompatibility, making them potential powerful PTT agents for tumor treatment via their NIR absorption properties. ${ }^{9-13}$

At present, the standard methods for measuring the efficiency of the PTT agents against tumor cells includes biochemical methods such as cytotoxicity assays and microscopy methods such as live and dead cell imaging assays. However, these methods could not provide dynamic information of cell death at single cell level or even at subcelluar level. Therefore, the subcellular processes of PTT triggered cell death still needs further investigation. Compared with the above-mentioned methods, fluorescence lifetime imaging microscopy (FLIM) technique has great advantages in monitoring multiple cell physiological processes, including cell growth and cell death, since FLIM can provide real-time images and quantitative subcellular information. ${ }^{14,15}$

In this study, we applied FLIM method to observe $\mathrm{MoS}_{2}$ nanosheet treated 4T1 cells in order to study the mitochondria damage during cell death. We used two types of $\mathrm{MoS}_{2}$ nanosheets, which are single layer nanosheets and few layer nanosheets (abbreviated as SL and FL, respectively), and obtained their photothermal conversion efficacy. Then, we confirmed their PTT efficacy on 4T1 cells under $808 \mathrm{~nm}$ laser irradiation. After the cell mitochondria were labeled with Rodamine123 (abbreviated as R123) dye, cells incubated with $\mathrm{MoS}_{2}$ nanosheets or control cells under $808 \mathrm{~nm}$ laser irradiation were respectively monitored by using FLIM technique. Finally, FLIM data were analyzed to evaluate the antitumor efficacy at single cell level.

\section{Methods}

\subsection{Chemicals}

Both of the $\mathrm{MoS}_{2}$ single layer nanosheets and few layer nanosheets were purchased from Nanjing XFNANO Materials Tech. Rodamine123 were purchased from Beyotime Biotechnology. Cell cytotoxicity kits (CCK-8) were obtained from Nanjing KeyGen Biotech. All the cell culture reagents (medium, antibiotics and fetal bovine serum) were obtained from Invitrogen.

\subsection{Material characterizations}

The morphology of the $\mathrm{MoS}_{2}$ nanosheets was obtained by a transmission electron microscopy (TEM) instrument (JEM-1230 CX, Jeol Ltd, Japan). The UV-Vis-NIR spectra of these nanosheets were recorded in a $10 \mathrm{~mm}$ path length cuvette on a spectrophotometer (UV1780, Shimadzu, Japan). The OD values in CCK-8 assays were determined by a microplate reader (Infinite M200, Tecan, Switzerland).

\subsection{Photothermal properties of the $\mathrm{MoS}_{2}$ nanosheets}

To evaluate the photothermal properties of the $\mathrm{MoS}_{2}$ nanosheets, a series of $\mathrm{MoS}_{2}$ aqueous solutions at different concentrations $(0,25,50,100,200$ or $400 \mu \mathrm{g} / \mathrm{mL}$ ) were prepared and deposited into cuvettes. Then the cuvettes were illuminated by a continuous-wave laser $(808 \mathrm{~nm})$ at a power density of $1 \mathrm{~W} / \mathrm{cm}^{2}$. The temperatures were continuously recorded by using a probe thermometer at every $30 \mathrm{~s}$ (for heating period) or every $1 \mathrm{~min}$ (for cooling period).

The photothermal conversion efficiency PTCE $(\eta)$ was calculated as mentioned before ${ }^{13,16,17}$ as follows:

$$
\eta=\frac{h A \Delta T_{\max }-Q s}{I\left(1-10^{-A 808}\right)} .
$$

In Eq. (1), $h$ represents the heat transfer coefficient. $\mathrm{A}$ is the container surface area $\left(1 \mathrm{~cm}^{2}\right) . \Delta T_{\max }$ is the maximum temperature change of the nanosheet solution at the corresponding time point. $Q s$ is defined as the heat loss due to the light absorbed by the container. $I$ refers to the incident power density of the $808 \mathrm{~nm}$ laser $\left(1 \mathrm{~W} / \mathrm{cm}^{2}\right)$ and A 808 is the absorbance value of the solution at $808 \mathrm{~nm}$. 
In order to get the $h A$ in Eq. (1), a parameter $\theta$ is introduced as shown in Eq. (2), which is defined as the ratio of $\Delta T$ to $\Delta T$ max.

$$
\theta=\frac{\Delta T}{\Delta T_{\max }} .
$$

$\Delta T$ stands for the temperature change during a cooling period. In addition, $h A$ is calculated by applying the linear time data from the cooling period versus $\ln \theta$.

\subsection{Cell culture and cytotoxicity assay}

4T1 cells (mouse breast cancer cells) were purchased from American Type Culture Collection (ATCC, USA). Cells were cultured in DMEM culture medium with $10 \%$ fetal bovine serum (FBS) and $1 \%$ penicillin-streptomycin at $37^{\circ} \mathrm{C}$ under a $5 \%$ $\mathrm{CO}_{2}$ and humidity condition.

In order to examine the biocompatibility of the $\mathrm{MoS}_{2}$ nanosheets, a standard CCK-8 cell assay was carried on 4T1 cells. Around 10,000 of cells were seeded in each well of 96 well plates and grown overnight. The next day, medium with different concentrations of the $\mathrm{MoS}_{2}$ nanosheets $(50,100$ or $200 \mu \mathrm{g} / \mathrm{mL}$ ) were added to the cells. Here, six replicates were applied for each condition. After $24 \mathrm{~h}$ incubation, the solutions were moved from cells and a mixture of $10 \mu \mathrm{L}$ of CCK-8 solution plus $100 \mu \mathrm{L}$ of fresh culture medium was added to each well, followed by another incubation for around 1$2 \mathrm{~h}$. At the end of the chromogenic reaction, the OD value at $450 \mathrm{~nm}$ wavelength of each well of the plates was recorded by using the microplate reader.

Next, the PTT efficiency of the $\mathrm{MoS}_{2}$ nanosheets was measured by the CCK-8 assay in a similar way. Briefly, 4T1 cells were seeded in 96-well plates and cultured overnight. Then, PBS or $\mathrm{MoS}_{2}$ nanosheets $(0,100$ or $200 \mu \mathrm{g} / \mathrm{mL})$ were added to the medium, and the cells were incubated in $5 \% \mathrm{CO}_{2}$ at $37^{\circ} \mathrm{C}$. After incubation, the $\mathrm{PBS}$ or $\mathrm{MoS}_{2}$ nanosheet treated samples (PTT groups) were illuminated under $808 \mathrm{~nm}$ laser irradiation $\left(1 \mathrm{~W} / \mathrm{cm}^{2}\right)$ for 5 mins, followed by the CCK- 8 assay at the same procedure mentioned above.

\subsection{FLIM experiments}

In order to obtain FLIM images, around 100,000 of cells were seeded in each $35 \mathrm{~mm}$ confocal dish. After an overnight incubation, the cells were labeled with R123 and then incubated infresh medium with either PBS or $\mathrm{MoS}_{2}$ nanosheets $(200 \mu \mathrm{g} / \mathrm{mL})$. These cell dishes were then moved onto the microscope stage and observed for 18 mins. The images were obtained at every 3 mins during this period. Next, the image acquisition was continuously performed at the same frequency while introducing the $808 \mathrm{~nm}$ laser irradiation $\left(1 \mathrm{~W} / \mathrm{cm}^{2}\right)$ onto the cells for another 15 mins.

All microscopic imaging and FLIM analyses were performed with a Nikon Eclipse confocal system mounted on an inverted microscope. This microscope was equipped with a Fianium whitelase Supercontinuum laser (output laser from 470 to $670 \mathrm{~nm}, 6 \mathrm{ps}$ pulse width, $80 \mathrm{MHz}$ repetition rate) for single photon excitation. A $63 \times$ oil immersion objective lens (NA 1.40) was used for all images. To obtain FLIM data, the time-correlated singlephoton counting (TCSPC) technique was carried out with a Becker \& Hickl SPC120 unit. In this study, the $514 \mathrm{~nm}$ excitation wavelength was selected to generate the fluorescence images of cells labeled with R123. A band-pass filter BP 535/30 was put in front of the PMT detector to acquire the specific signals. All FLIM images were acquired at $256 \times 256$ pixels and the collection time for one image was around $30 \mathrm{~s}$. After image collection, the fluorescence lifetime image data was analyzed by using SPCImage (Becker \& Hickl) software as described in our former studies, ${ }^{18-20}$ providing the fluorescence decay signal of each image and lifetimes of the selected regions.

Next, to evaluate the photostability of R123 dye under NIR light irradiation, we carried out the following experiments. Briefly, the R123 solution $(50 \mu \mathrm{M})$ or the cells labeled with R123 were prepared in confocal dishes. Then the dishes were moved onto the microscope stage and observed for 18 mins. The images were obtained at every 3 mins during this period and the photon numbers collected in $1 \mathrm{~min}$ were counted at each timepoint. After the $808 \mathrm{~nm}$ laser irradiation $\left(1 \mathrm{~W} / \mathrm{cm}^{2}\right)$ was turned on, the photon-counting image acquisition was continuously carried out for extra 15 mins.

In addition, we used FLIM technique to investigate the parameters that might influence the fluorescence lifetime of R123 dye. The R123 dye samples were prepared in a series of solutions $(100 \mu \mathrm{M})$ with different $\mathrm{pH}$ values or with different viscosities. Then these two series of R123 dye solutions were measured with FLIM system, providing images for data analysis. 


\section{Results and Discussion}

\subsection{Characterization of $\mathrm{MoS}_{2}$ nanosheets}

In this study, both $\mathrm{MoS}_{2}$ nanosheets are stored in aqueous solutions. As shown in the TEM image (Figs. 1(a) and 1(b)), a general view of the $\mathrm{MoS}_{2}$ nanosheets showed that the lateral sizes of $\mathrm{MoS}_{2} \mathrm{SL}$ nanosheets and FL nanosheets are both in the range from around 50 to $200 \mathrm{~nm}$, which is a suitable size range for efficient endocytosis in the applications of nanoparticles into biological fields. ${ }^{21,22}$

Moreover, the absorption range of both of the two types of $\mathrm{MoS}_{2}$ nanosheets with the gradient concentrations $(25,50,100,200$ or $400 \mu \mathrm{g} / \mathrm{mL})$ expand to the NIR region from 700 to $900 \mathrm{~nm}$

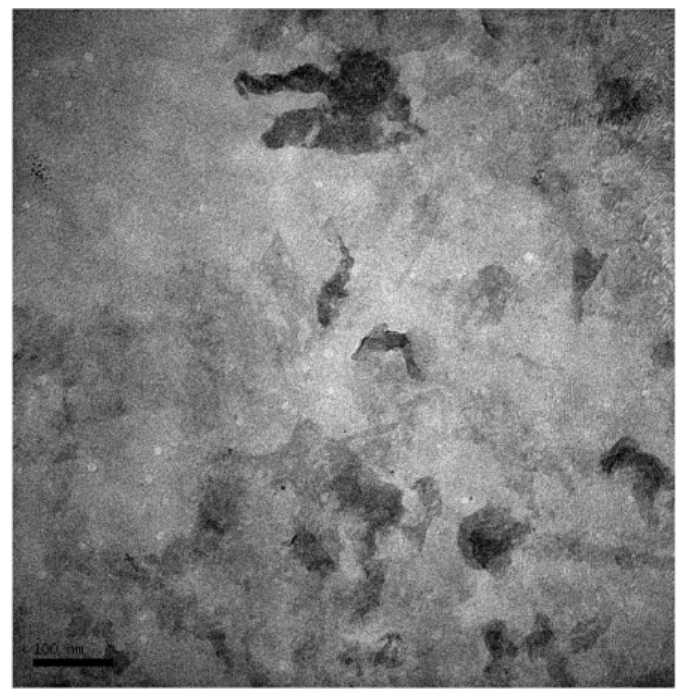

(a)

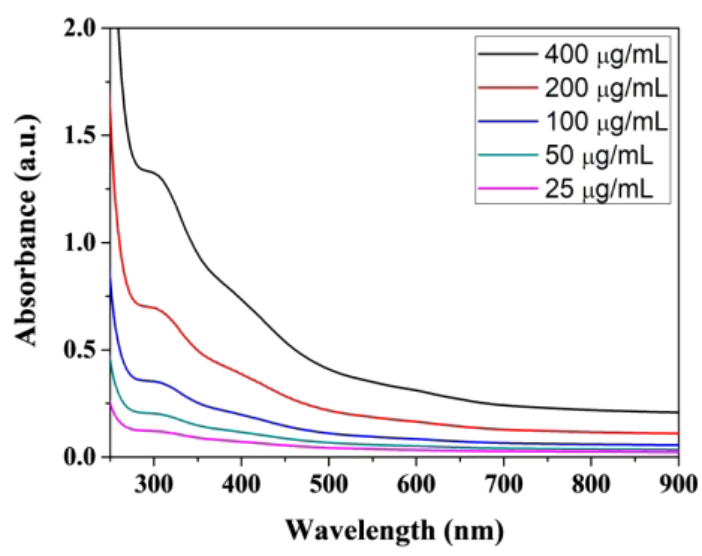

(c)
(Figs. 1(c) and 1(d)), which is very similar to the results reported before. ${ }^{9-13}$ This range is compliant with an $808 \mathrm{~nm}$ laser in the further investigation on their photothermal properties.

\subsection{Photothermal properties of $\mathrm{MoS}_{2}$ nanosheets}

In order to examine the photothermal properties of $\mathrm{MoS}_{2}$ SL nanosheets and FL nanosheets, we recorded the temperature trends of the aqueous solutions with a series of concentrations $(0,50,100$, and $200 \mu \mathrm{g} / \mathrm{mL}$ ) that were exposed to an $808-\mathrm{nm}$ laser irradiation $\left(1 \mathrm{~W} / \mathrm{cm}^{2}\right)$ for 5 mins.

As shown in Figs. 2(a) and 2(b), the solution temperatures rapidly increase in $5 \mathrm{mins}$, and the

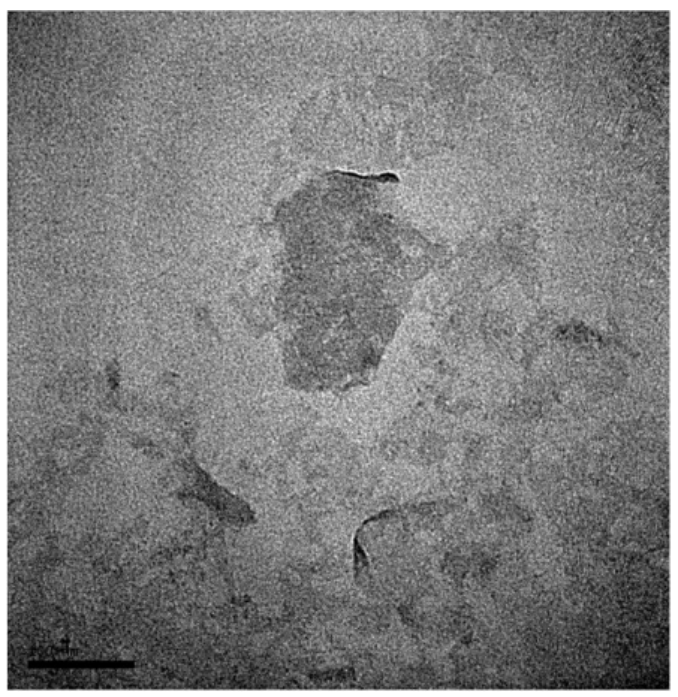

(b)

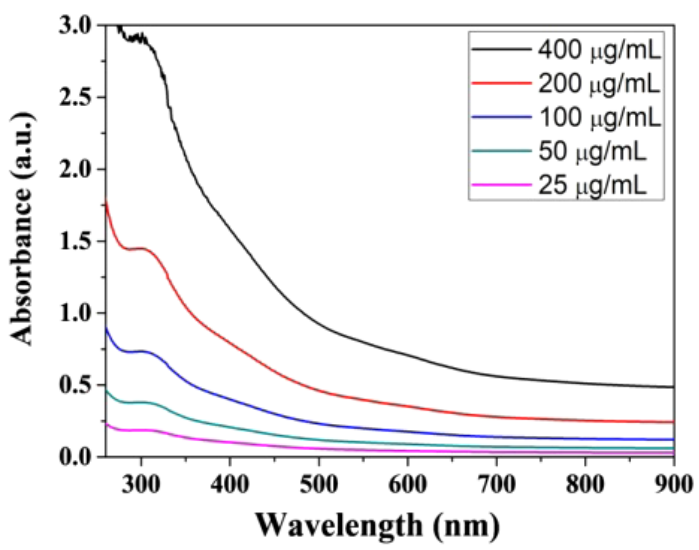

(d)

Fig. 1. (a)-(b) TEM images of $\mathrm{MoS}_{2}$ single-layer nanosheets (a) and few-layer nanosheets (b), respectively. Scalebar: $100 \mathrm{~nm}$. (c) and (d) Absorbance spectra of $\mathrm{MoS}_{2}$ SL (c) and FL (d) nanosheets with the gradient concentrations, respectively. 


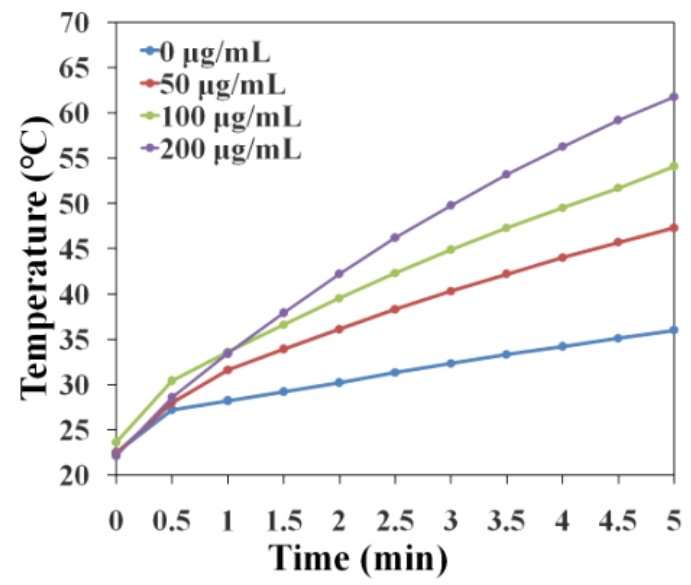

(a)

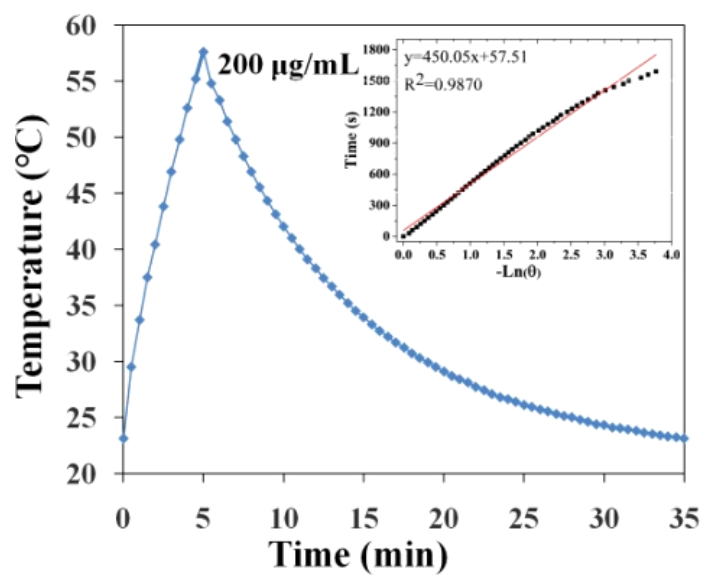

(c)

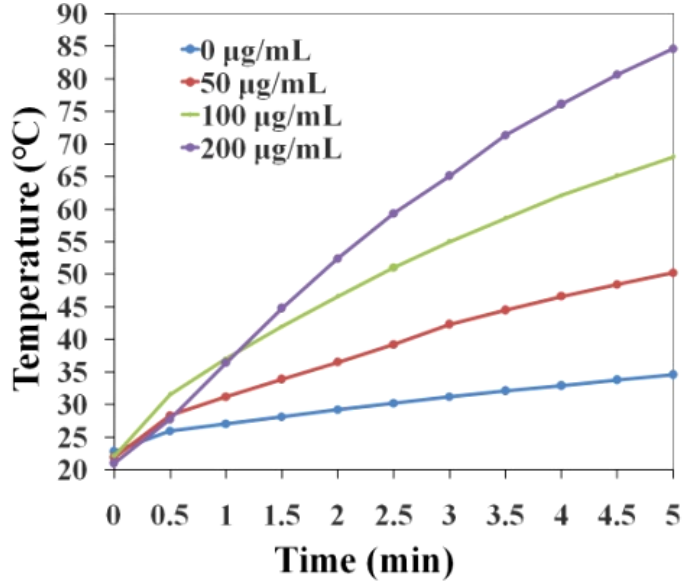

(b)

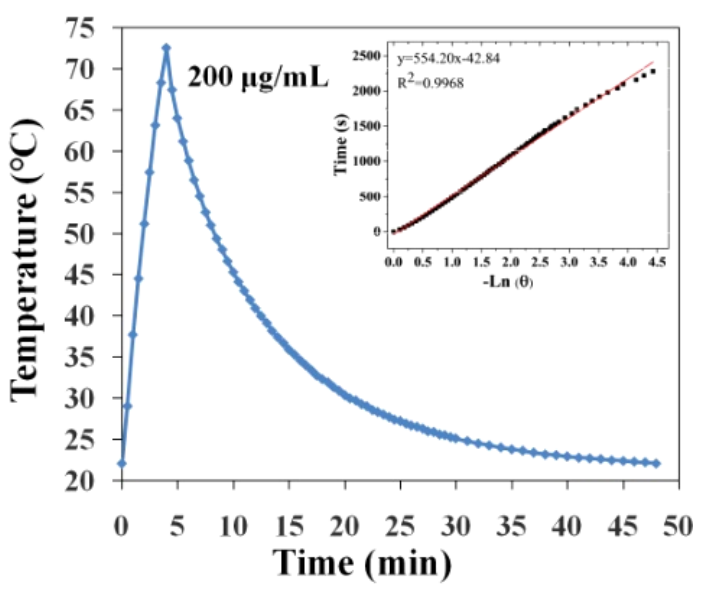

(d)

Fig. 2. (a)-(b) Heating curves of $\mathrm{MoS}_{2}$ SL nanosheets (a) or $\mathrm{MoS}_{2}$ FL nanosheets (b) aqueous solution with a series of concentrations during irradiation $\left(808 \mathrm{~nm}, 1 \mathrm{~W} / \mathrm{cm}^{2}\right)$. (c)-(d) The temperature profile of the $\mathrm{MoS}_{2}$ SL nanosheets $(\mathrm{c})$ or $\mathrm{MoS}_{2} \mathrm{FL}$ nanosheets (d) under irradiation for 5 mins $\left(808 \mathrm{~nm}, 1 \mathrm{~W} / \mathrm{cm}^{2}\right)$ and the following cooling period (turning off the laser). The insets are the relationship between $-\operatorname{Ln}(\theta)$ and time of $\mathrm{MoS}_{2}$ SL nanosheets (c) or MoS $\mathrm{ML}_{2}$ nanosheets (d), which were calculated from the cooling curves, respectively.

slopes are positively associated with the increased concentrations of nanomaterials. For example, the temperature of the $\mathrm{MoS}_{2} \mathrm{SL}$ nanosheet solution $(200 \mu \mathrm{g} / \mathrm{mL})$ dramatically increased from around $23.0^{\circ} \mathrm{C}$ to $61.8^{\circ} \mathrm{C}$ in 5 mins, achieving an excellent temperature high enough to an irreversible ablation to the tumor cells. ${ }^{23}$ Under the same condition, the temperature change of the $\mathrm{MoS}_{2}$ FL nanosheet solution $(200 \mu \mathrm{g} / \mathrm{mL})$ exhibited even a greater increase to around $80^{\circ} \mathrm{C}$ after laser irradiation for 5 mins, which indicates the higher photothermal transduction efficacy of $\mathrm{MoS}_{2}$ FL than that of $\mathrm{MoS}_{2}$ SL nanosheets.

To further investigate the photothermal transduction ability of these two types of nanosheet aqueous solution, we measured the temperature profiles of each solution with a concentration of $200 \mu \mathrm{g} / \mathrm{mL}$ under a continuous laser irradiation $\left(1 \mathrm{~W} / \mathrm{cm}^{2}\right)$ for $5 \mathrm{mins}$, consequently followed by a natural cooling period till room temperature. The temperature profile results were shown as in Figs. 2(c) and 2(d). These data were analyzed according to the reported method, resulting in the photothermal conversion efficiency $(\eta)$ of the $\mathrm{MoS}_{2}$ SL and the $\mathrm{MoS}_{2}$ FL nanosheets, which could achieve $17.4 \%$ and $24.8 \%$, respectively, similar to that of previously reported $\mathrm{MoS}_{2}$ nanosheets $(24.37 \%$ or $26.7 \%){ }^{9,13}$ Such suitable photothermal conversion capabilities indicate that $\mathrm{MoS}_{2}$ nanosheets could offer great advantages for PTT. 


\subsection{Photothermal therapy in vitro}

In order to evaluate the potential PTT application abilities of $\mathrm{MoS}_{2}$ SL or FL nanosheets, we carried out cell cytotoxicity assay as wells as PTT effect assay on $4 \mathrm{~T} 1$ cells. Firstly, the potential toxicity of $\mathrm{MoS}_{2}$ SL or $\mathrm{MoS}_{2}$ FL was measured with a CCK-8 assay as the manufactory's instructions. Experimental data showed that there was little cytotoxicity for either $\mathrm{MoS}_{2} \mathrm{SL}$ or FL nanosheets after $24 \mathrm{~h}$ incubation, even at the highest concentration $200 \mu \mathrm{g} / \mathrm{mL}$ (Fig. 3(a)), indicating their good biological compatibility.

In addition, we examined the effect of the PTT of $\mathrm{MoS}_{2}$ SL or FL nanosheets in $4 \mathrm{~T} 1$ cells by an exposure to the $808 \mathrm{~nm}$ laser irradiation. As presented in Figs. 3(b) and 3(c), in the PBS, only nanosheets, or only NIR $\left(808 \mathrm{~nm}, 1 \mathrm{~W} / \mathrm{cm}^{2}, 5 \mathrm{mins}\right)$ treated group, all the cell viabilities reached more than $90 \%$, suggesting negligible damage effects. By contrast, in the $\mathrm{MoS}_{2}$ SL or FL nanosheets plus NIR irradiation group, more than $80 \%$ of the cells were killed due to the nanosheet induced PTT effects, which were consistent with former studies. ${ }^{9,17}$

\subsection{FLIM data acquisition and analysis}

Finally, we performed FLIM experiments for measuring the PTT effects of $\mathrm{MoS}_{2}$ SL or FL nanosheets on $4 \mathrm{~T} 1$ cells to acquire dynamic subcellular information. In order to monitor the local molecular environment changes in live $4 \mathrm{~T} 1$ cells, the fluorescent dye R123 was used as a mitochondria localization marker in this process. ${ }^{24,25}$ During each experiment, around 10-20 of live 4T1 cells were selected for continuously monitoring the fluorescence lifetime of R123 in cell mitochondria. The fluorescence lifetime image of these cells was obtained for every $3 \mathrm{mins}$ in the first period (no irradiation). Then an $808 \mathrm{~nm}$ laser was turned on to

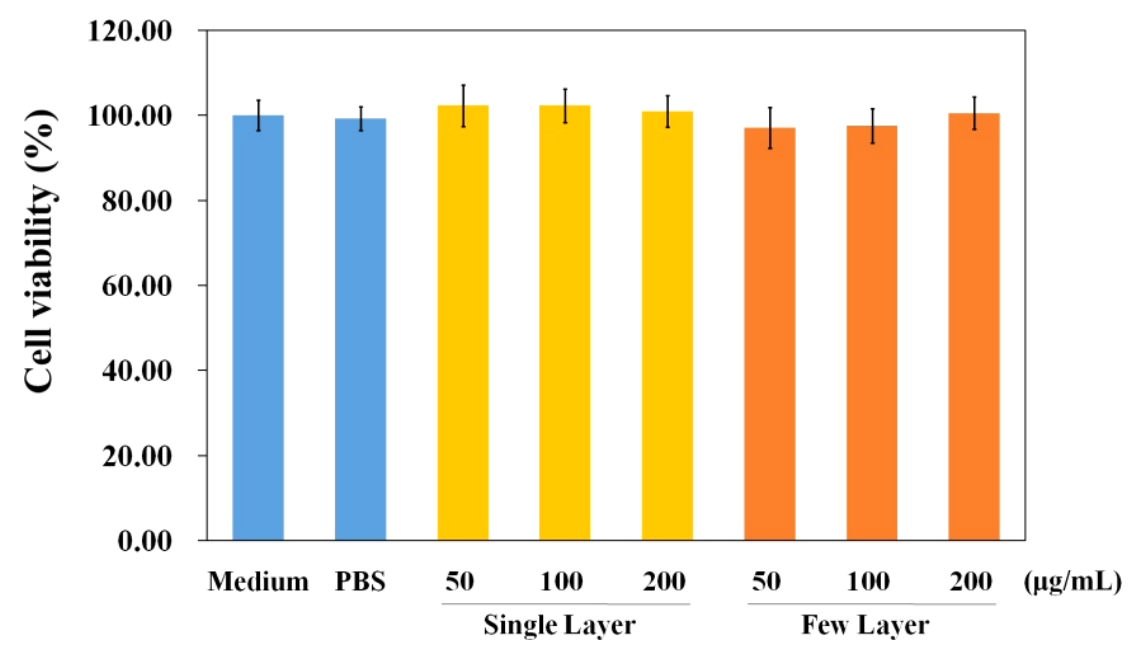

(a)

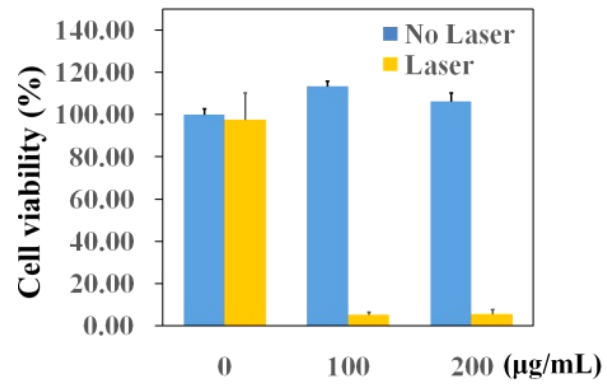

(b)

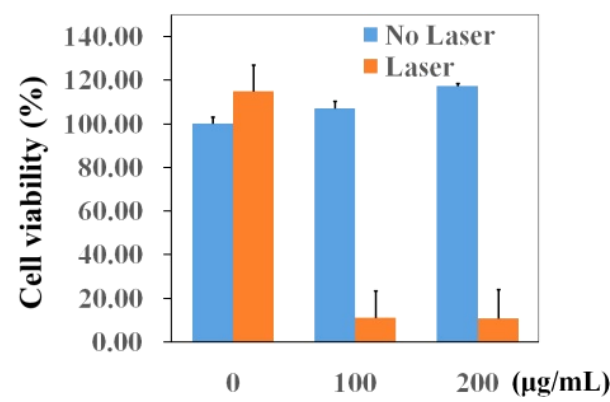

(c)

Fig. 3. (a) Cell viability of $4 \mathrm{~T} 1$ cells after incubation with different concentrations of $\mathrm{MoS}_{2} \mathrm{SL}$ or MoS $\mathrm{S}_{2} \mathrm{FL}$ nanosheets after $24 \mathrm{~h}$ incubation. (b)-(c) Cell viability of $4 \mathrm{~T} 1$ cells treated with $\mathrm{MoS}_{2} \mathrm{SL}$ (b) or $\mathrm{MoS}_{2} \mathrm{FL}$ (c) nanosheets with or without laser irradiation $\left(808 \mathrm{~nm}, 1 \mathrm{~W} / \mathrm{cm}^{2}, 5 \mathrm{mins}\right)$. Data are shown as mean $\pm \mathrm{SEM}$. 


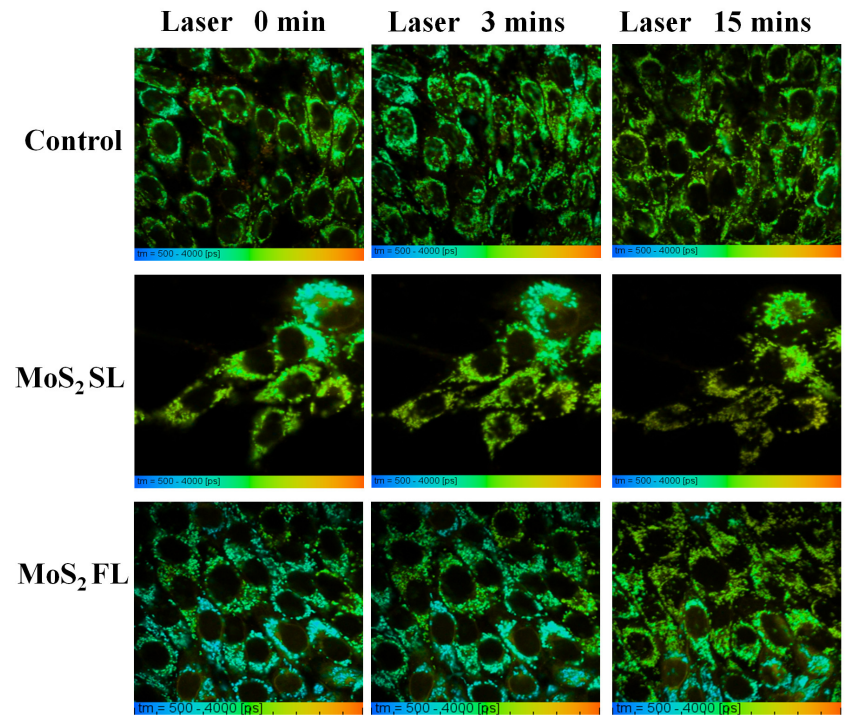

(a)

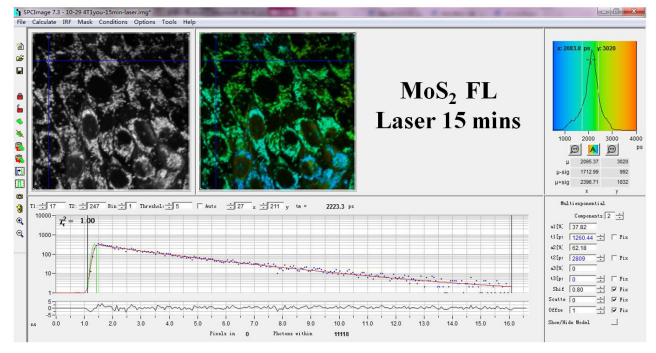

(b)

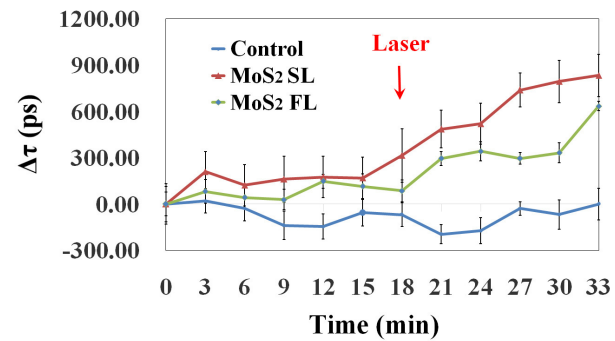

(c)

Fig. 4. (a) The fluorescence lifetime images of the $4 \mathrm{~T} 1$ cells treated with PBS (as control), MoS $\mathrm{SL}_{2}$ or MoS 2 FL nanosheets under NIR laser irradiation at $0 \mathrm{~min}, 3 \mathrm{mins}$ or $15 \mathrm{mins}\left(808 \mathrm{~nm}, 1 \mathrm{~W} / \mathrm{cm}^{2}\right)$. (b) One representative raw data from the cells treated with $\mathrm{MoS}_{2}$ FL nanosheets under NIR laser irradiation at 15 mins. The insets in the upper-left region are the fluorescence intensity and the fluorescence lifetime images, respectively. The inset in the upper-right corner shows the histogram of lifetime distribution of the fluorescent dye. The inset in the lower part represents the decay curve, in which the $x$ axis means the counting time while the $y$ axis shows the counted photon number. (c) Changes of the fluorescence lifetimes of R123 dyes in the cells treated with PBS (as control), $\mathrm{MoS}_{2}$ SL or $\mathrm{MoS}_{2}$ FL nanosheets under laser irradiation $\left(808 \mathrm{~nm}, 1 \mathrm{~W} / \mathrm{cm}^{2}\right)$.

irritate the cells at $1 \mathrm{~W} / \mathrm{cm}^{2}$. We recorded the fluorescence lifetime of R123 for 18 mins and then kept recording for another 15 mins after laser on.

Figure 4(a) were a series of fluorescence lifetime images of typical 4T1 cells during laser irradiation, representing the R123 fluorescence lifetime distributions of the dye in the cell mitochondria. Figure 4(b) was one representative raw result that collected the cells treated with $\mathrm{MoS}_{2}$ FL nanosheets under NIR laser irradiation at 15 mins. This figure includes the fluorescence intensity image which indicates suitable signal intensity, the fluorescence lifetime image and the histogram of the lifetime distribution of the fluorescent R123 dye in the whole image. The inset in the lower part provides a decay curve for calculating the fluorescence lifetime. As shown in Fig. 4(c), the R123 fluorescence lifetime from cells treated with both types of $\mathrm{MoS}_{2}$ nanosheet showed slight fluctuations during the first 18 mins before laser on, indicating the nanosheet incubation had no effect on the fluorescence lifetime values. From the initial of laser irradiation, the fluorescence lifetimes of R123 in cells treated with both $\mathrm{MoS}_{2}$ nanosheets significantly increased in an irreversible way. By contrast, the fluorescence lifetime of the same dye in the control cells remained constant. These above data showed that under physiological conditions, the fluorescence lifetimes of R123 in healthy $4 \mathrm{~T} 1$ cells could keep constant with random fluctuation. The values of such fluctuation are usually in the range of 50 $200 \mathrm{ps}$. The induction of NIR laser exposure onto nanosheet treated $4 \mathrm{~T} 1$ cells lead to around $500 \mathrm{ps}$ increases the obtained fluorescence lifetimes suggesting the significant correlation between the cell death and the PTT effects.

Moreover, the photostability of R123 were tested by exposing the R123 solution or the cells labeled with R123 under NIR laser irradiation. Both in vitro and in vivo results are shown in Figs. 5(a) and 5(b), respectively. The collected photon number of the in vitro sample keeps almost constant while the photon number from in vivo sample maintains around $90 \%$ after laser explosion, suggesting the satisfactory photostability of R123 through the whole experiment processes.

To further understand the potential microenvironment parameters that might influence the fluorescence lifetimes of R123, the R123 dye samples in different solutions were then measured with FLIM 


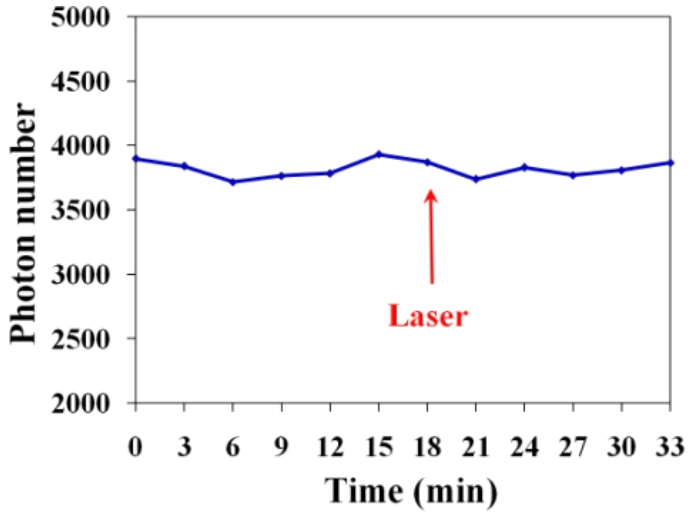

(a)

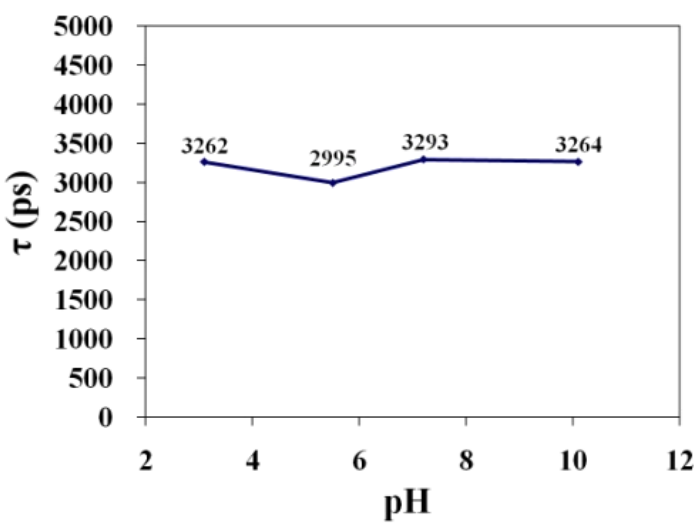

(c)

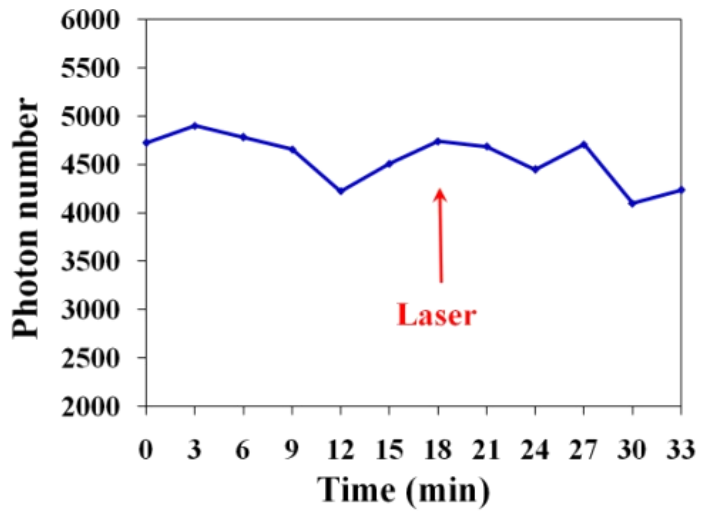

(b)

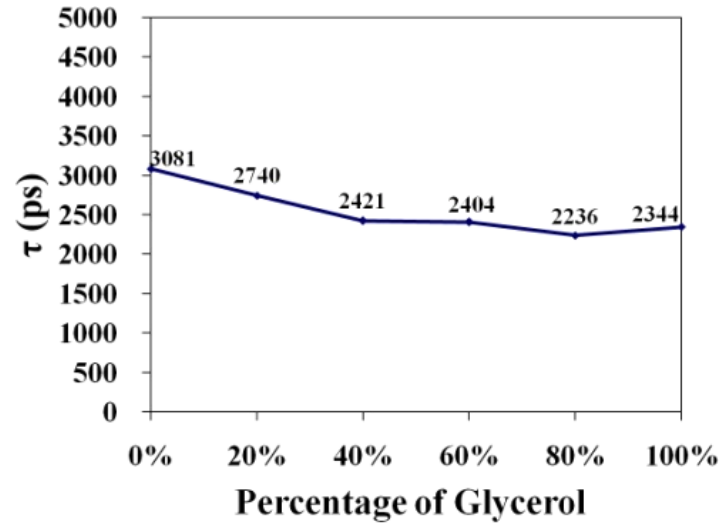

(d)

Fig. 5. (a)-(b) The trends of photon numbers collected in 1 min from samples of the R123 solution in vitro (a) or of the R123 in cells (b) under NIR laser irradiation $\left(808 \mathrm{~nm}, 1 \mathrm{~W} / \mathrm{cm}^{2}\right)$. (c)-(d) The fluorescence lifetimes of R123 dye in PBS solutions containing different $\mathrm{pH}$ values $(\mathrm{c})$ or in glycerol-water solutions containing different viscosities $(\mathrm{d})$.

system. The images were then obtained and analyzed, providing the data in Figs. 5(c) and 5(d). The fluorescence lifetimes of R123 fluctuated slightly at different $\mathrm{pH}$ conditions (Fig. 5(c)). By contrast, as shown in the Fig. 5(d), the R123 decreased as the percentage of glycerol in glycerol-water solutions increased from $0 \%$ to $80 \%$, indicating that the fluorescence lifetime of R123 might be sensitive to the change of viscosity.

R123 has been considered as a satisfactory livecell probe for decades, which could target mitochondria due to its property of probing transmembrane potential. ${ }^{24,25}$ In this work, when the $4 \mathrm{~T} 1$ cells were killed during PTT procedures, the transmembrane potential of mitochondria might decline upon cells death, leading to the release of R123 dye from mitochondria into cytoplasm. As a result, the altered microenviroment might cause the changes of the R123 fluorescence lifetime. Therefore, these results suggest that FLIM could provide realtime quantitative data in monitoring PTT triggered cancer cell death.

\section{Summary}

In this study, $\mathrm{MoS}_{2} \mathrm{SL}$ and FL nanosheets have been used for PTT in 4T1 cells. The NIR photothermal conversion efficiency of $\mathrm{MoS}_{2} \mathrm{SL}$ and FL nanosheets reach the satisfactory values, ensuring them strong capabilities as powerful PTT agents. The CCK-8 data show that both the nanosheets have little toxicity and great cell killing efficacy to $4 \mathrm{~T} 1$ cells. In addition, FLIM techniques are used to monitor the local environment changes of the mitochondria compartments in live $4 \mathrm{~T} 1$ cells. The significant fluorescence lifetime changes in mitochondria of the nanosheet treated cancer cells have been proved to be quantitative measurement 
methods for representing PTT induced cell death in a non invasive way. Currently, the organelletargeting and image-guided therapy has attracted more and more research interests. ${ }^{26}$ Therefore, this work is expected to expand the utility of FLIM for specific subcellular microenvironments into evaluating and examining the development of novel tumor thermo-chemo therapies.

\section{Conflict of Interest}

The authors have declared that no competing interest exists.

\section{Acknowledgments}

This work was supported by the National Key R\&D Program of China (2018YFC0910602), the National Natural Science Foundation of China (Grant Nos. 31771584/61775145/61605121, 61620106016/61525503/61835009/81727804), Guangdong Natural Science Foundation Innovation Team (2014A030312008), and Shenzhen Basic Research Project (JCYJ20170818100153423/JCYJ201704 12110212234/JCYJ20160328144746940/JCYJ2017 0412105003520/JCYJ20170302142902581), Science Foundation of SZU (Grant No. 000193).

\section{References}

1. C. J. Lord, A. Ashworth, "The DNA damage response and cancer therapy," Nature 481, 287-294 (2012).

2. W. Landier, "Ototoxicity and cancer therapy," Cancer 122, 1647-1658 (2016).

3. S. Vijayakumar, S. Selvakumar, Y. Chen-Sheng, "Near-infrared light-responsive nanomaterials in cancer therapeutics," Chem. Soc. Rev. 43, 6254-6287 (2014).

4. H. Li, P. Wang, Y. Deng, M. Zeng, Y. Tang, W. H. Zhu, Y. Cheng, "Combination of active targeting, enzyme-triggered release and fluorescent dye into gold nanoclusters for endomicroscopy-guided photothermal/photodynamic therapy to pancreatic ductal adenocarcinoma," Biomaterials 139, 30-38 (2017).

5. J. J. Hu, Y. J. Cheng, X. Z. Zhang, "Recent advances in nanomaterials for enhanced photothermal therapy of tumors," Nanoscale 10, 22657-22672 (2018).

6. Y. Chen, Z. Fan, Z. Zhang, W. Niu, C. Li, N. Yang, B. Chen, H. Zhang, "Two-dimensional metal nanomaterials: Synthesis, properties, and applications," Chem. Soc. Rev. 118, 6409-6455 (2018).

7. T. Fan, Y. Zhou, M. Qiu, H. Zhang, "Black phosphorus: A novel nanoplatform with potential in the field of bio-photonic nanomedicine," JIOHS 11, 1830001 (2018).

8. C. Murugan, V. Sharma, R. K. Murugan, G. Malaimegu, A. Sundaramurthy, "Two-dimensional cancer theranostic nanomaterials: Synthesis, surface functionalization and applications in photothermal therapy," J. Control. Release 299, 1-20 (2019).

9. W. Yin, L. Yan, J. Yu, G. Tian, L. Zhou, X. Zheng, X. Zhang, Y. Yong, J. Li, Z. Gu, "High-throughput synthesis of single-layer $\mathrm{MoS}_{2}$ nanosheets as a nearinfrared photothermal-triggered drug delivery for effective cancer therapy," ACS Nano. 8, 6922-6933 (2014).

10. T. Liu, C. Wang, X. Gu, H. Gong, L. Cheng, X. Shi, L. Feng, B. Sun, Z. Liu, "Drug delivery with PEGylated $\mathrm{MoS}_{2}$ nano-sheets for combined photothermal and chemotherapy of cancer," Adv. Mater. 26, 3433-3440 (2014).

11. T. Shao, J. Wen, Q. Zhang, Y. Zhou, L. Liu, L. Yuwen, Y. Tian, Y. Zhang, W. Tian, Y. Su, Z. Teng, G. Lu, J. Xu, "NIR photoresponsive drug delivery and synergistic chemo-photothermal therapy by monodispersed- $\mathrm{MoS}_{2}$-nanosheets wrapped periodic mesoporous organosilicas," J. Mater. Chem. B 4, 7708-7717 (2016).

12. L. Kong, L. Xing, B. Zhou, L. Du, X. Shi, "Dendrimer-modified $\mathrm{MoS}_{2}$ nanoflakes as a platform for combinational gene silencing and photothermal therapy of tumors," ACS Appl. Mater. Interf. 9, 15995-16005 (2017).

13. S. Gao, H. Zhou, S. Cui, H. Shen, "Bottom-up synthesis of $\mathrm{MoS}_{2}$ nanospheres for photothermal treatment of tumors," Photochem. Photobiol. Sci. 17, 1337-1345 (2018).

14. S. M. Levchenko, A. Pliss, J. Qu, "Fluorescence lifetime imaging of fuorescent proteins as an effective quantitative tool for noninvasive study of intracellular processes," JIOHS 11, 1730009 (2018).

15. S. Suarasan, A. M. Craciun, E. Licarete, M. Focsan, K. Magyari, S. Astilean, "Intracellular dynamic disentangling of doxorubicin release from luminescent nanogold carriers by fluorescence lifetime imaging microscopy (FLIM) under two-photon excitation," ACS Appl. Mater. Interf. 11, 7812-7822 (2019).

16. D. K. Roper, W. Ahn, M. Hoepfner, "Microscale heat transfer transduced by surface Plasmon resonant gold nanoparticles," J. Phys. Chem. C Nanomater Interf. 111, 3636-3641 (2007). 
17. Q. Wu, Y. Lin, F. Wo, Y. Yuan, Q. Ouyang, J. Song, J. Qu, K. T. Yong, "Novel magneticluminescent janus nanoparticles for cell labeling and tumor photothermal therapy," Small 13, 1701129 (2017).

18. A. Pliss, X. Peng, L. Liu, A. Kuzmin, Y. Wang, J. Qu, Y. Li, P. N. Prasad, "Single cell assay for molecular diagnostics and medicine: Monitoring intracellular concentrations of macromolecules by two-photon fluorescence lifetime imaging," Theranostics 5, 919-930 (2015).

19. S. Zhou, X. Peng, H. Xu, Y. Qin, D. Jiang, J. Qu, H. Y. Chen, "Fluorescence lifetime-resolved ionselective nanospheres for simultaneous imaging of calcium ion in mitochondria and lysosomes," Anal. Chem. 90, 7982-7988 (2018).

20. A. Pliss, S. M. Levchenko, L. Liu, X. Peng, T. Y. Ohulchanskyy, I. Roy, A. N. Kuzmin, J. Qu, P. N. Prasad, "Cycles of protein condensation and discharge in nuclear organelles studied by fluorescence lifetime imaging," Nat. Commun. 10, 455 (2019).

21. W. Jiang, B. Y. Kim, J. T. Rutka, W. C. Chan, "Nanoparticle-mediated cellular response is sizedependent," Nat. Nanotechnol. 3, 145-150 (2008).

22. H. Cabral, Y. Matsumoto, K. Mizuno, Q. Chen, M. Murakami, M. Kimura, Y. Terada, M. R. Kano,
K. Miyazono, M. Uesaka, N. Nishiyama, K. Kataoka, "Accumulation of sub-100 nm polymeric micelles in poorly permeable tumours depends on size," Nat. Nanotechnol. 6, 815-823 (2011).

23. S. N. Goldberg, G. S. Gazelle, P. R. Mueller, "Thermal ablation therapy for focal malignancy: A unified approach to underlying principles, techniques, and diagnostic imaging guidance," $A m$. J. Roentgenol. 174, 323-331 (2000).

24. R. K. Emaus, R. Grunwald, J. J. Lemasters, "Rhodamine 123 as a probe of transmembrane potential in isolated rat-liver mitochondria: Spectral and metabolic properties," Biochim. Biophys. Acta. 850, 436-448 (1986).

25. H. Schneckenburger, K. Stock, M. Lyttek, W. S. Strauss, R. Sailer, "Fluorescence lifetime imaging (FLIM) of rhodamine 123 in living cells," Photochem. Photobiol. Sci. 3, 127-131 (2004).

26. J. Zhang, Q. Wang, Z. Guo, S. Zhang, C. Yan, H. Tian, W. H. Zhu, "High-fidelity trapping of spatialtemporal mitochondria with rational design of aggregation-induced emission probes," Adv. Funct. Mater. 29, 1808153 (2019). 